\title{
Randomization beats Second Price as a Prior-Independent Auction
}

\author{
$\mathrm{Hu} \mathrm{Fu}^{*} \quad$ Nicole Immorlica ${ }^{\dagger} \quad$ Brendan Lucier $^{\ddagger} \quad$ Philipp Strack $^{\S}$
}

April 26, 2018

\begin{abstract}
Designing revenue optimal auctions for selling an item to $n$ symmetric bidders is a fundamental problem in mechanism design. Myerson (1981) shows that the second price auction with an appropriate reserve price is optimal when bidders' values are drawn i.i.d. from a known regular distribution. A cornerstone in the prior-independent revenue maximization literature is a result by Bulow and Klemperer (1996) showing that the second price auction without a reserve achieves $(n-1) / n$ of the optimal revenue in the worst case.

We construct a randomized mechanism that strictly outperforms the second price auction in this setting. Our mechanism inflates the second highest bid with a probability that varies with $n$. For two bidders we improve the performance guarantee from 0.5 to 0.512 of the optimal revenue. We also resolve a question in the design of revenue optimal mechanisms that have access to a single sample from an unknown distribution. We show that a randomized mechanism strictly outperforms all deterministic mechanisms in terms of worst case guarantee.
\end{abstract}

\section{Introduction}

Designing revenue optimal auctions to sell an item to symmetric bidders is one of the most fundamental problems in optimal mechanism design. This problem was solved by Myerson (1981): if the buyers' valuations for the item are independently drawn from a distribution which is known to the seller, the revenue-maximizing mechanism is a second price auction (SPA) with a reserve price. Notably, to determine the reserve price and implement the optimal auction the seller needs to know the valuation distribution. Since setting the reserve price too high might leave the seller with no revenue, the design of auctions with good revenue guarantee when the prior information is unavailable, or very costly to learn, is of high economic relevance.

A first answer to this question was given by Bulow and Klemperer (1996), who show that for regular 1 distributions a second price auction with a reserve price of zero guarantees the seller at least a $1-1 / n$ fraction of the optimal revenue, where $n$ is the number of bidders. In particular, this yields at least half of the optimal revenue whenever there are at least two bidders in the auction. Note that this auction is prior-independent, in the sense that it does not depend on the prior distribution from which valuations are drawn. Moreover, it is clear that this is the best possible

\footnotetext{
*Microsoft Research New England, hufu@microsoft.com

${ }^{\dagger}$ Microsoft Research New England, nicimm@gmail.com

${ }^{\ddagger}$ Microsoft Research New England, brlucier@microsoft.com

$\S$ University of California Berkeley, pstrack@berkeley.edu

${ }^{1} \mathrm{~A}$ distribution is regular if the virtual valuation is non-decreasing. This assumption, which is standard in the mechanism design literature, will be discussed in Section 2
} 
way to set a deterministic reserve price: any reserve greater than zero yields zero revenue when all bidders valuations fall below it, so no deterministic positive reserve can guarantee the seller a higher share of the optimal revenue. In other words, the fixed reserve price that guarantees the seller the highest share of the optimal revenue, against all regular distributions, equals zero. In fact, with the characterization developed by Myerson, it is not hard to show that no symmetric deterministic auction can improve upon Bulow and Klemperer's guarantee of half of the optimal revenue (for two bidders).

This paper gives the first mechanism that outperforms the second price auction in Bulow and Klemperer (1996)'s setting, by way of making use of randomization. This mechanism, which we call bid inflation, works as follows: with a fixed probability $1-\epsilon<1$, the mechanism runs a SPA with a reserve price of zero; i.e., each bidder gets the object if and only if his valuation is above all other bidders' valuations. With the remaining probability $\epsilon>0$, the mechanism allocates the object to the bidder with the highest valuation if and only if his valuation is greater than $(1+\delta)>1$ times the valuation of any other bidder, otherwise the object is unallocated.

The idea behind bid inflation is based upon a refined analysis of the result by Bulow and Klemperer (1996). Our analysis distinguishes two types of distributions: those for which the optimal reserve price is high, and those for which it is low. A reserve price is considered high (low) if the probability that the valuation exceeds it is low (high). We first show that whenever the optimal reserve price is sufficiently low, the second price auction without reserve obtains strictly more than a $(1-1 / n)$ fraction of the optimal revenue. This suggests that one should design a mechanism which works well for distributions with high reserve prices. An analysis à la Myerson suggests that one should try not to allocate to bids below the optimal reserve price and only allocate to the bids above it (even without knowing the optimal reserve). Whereas inflating the second highest bid will correctly keep the item unsold when the highest bid is not high enough, this will also inevitably lose revenue when the highest bid is high but the second highest bid is not far below. The key to our analysis is to show that for distributions with high reserves, the loss from the latter scenario does not offset the gain from the former one. This in turn relies on a technical lemma which controls the quantile, i.e., the relative standing in a distribution, as a valuation changes in a regular distribution. With this analysis for the inflated second price auction in hand, one can then show via a continuity argument that a probabilistic mixture of the inflated auction and the second price auction without a reserve price guarantees a better approximation for all distributions.

While the main point of this paper is to demonstrate that there exist randomized mechanisms that outperform the second-price auction in a worst case analysis over regular distributions, we also quantify the improvement in the two bidder case. For two bidders, a second price auction with zero reserve price guarantees the seller half of the optimal revenue. We show that the bid inflation mechanism, where the higher valuation bidder receives the object with probability $85 \%$ if his valuation is below twice the other bidder's valuation, guarantees a revenue of at least $51.2 \%$ of the optimal revenue. Thus, for two players the bid inflation mechanism generates a $2.4 \%$ higher revenue guarantee in the worst case scenario.

Finally, we also consider a variant of the prior-independent setting, in which the seller gains a small amount of information about the prior distribution in the form of a sample. This sampling model has gained recent attention in the computer science literature. In a spirit similar to the result of Bulow and Klemperer, it is known that running a second-price auction, with a reserve set equal to the sampled value, guarantees at least $1-\frac{1}{n+1}$ fraction of the optimal revenue for 
arbitrary regular distributions; when there is only a single bidder, posting the sampled value as a take-it-or-leave-it price gives therefore half of the optimal revenue (Dhangwatnotai et al., 2014). It is also known that no deterministic pricing method can improve upon this guarantee for a single bidder (Huang et al., 2014). However, we show how to construct a randomized mechanism which improves upon this revenue guarentee and obtains more than half of the optimal revenue for a single bidder. The construction is of a flavour similar to the bid-inflation mechanism: it offers the sampled value as a take-it-or-leave-it price, but sometimes either inflates the value or shades it down before using it.

Dhangwatnotai et al. (2014) uses the single sample technique to construct a prior-independent auction for settings where bidders' values are independent draws from non-identical regular distributions but each distribution gives rise to at least two bidders' values. Our improvement for the single bidder single sample scenario immediately implies a better prior-independent auction in this setting.

\section{Related Literature}

As the bid inflation mechanism is independent of the distribution of valuations it follows the doctrine propagated by Wilson (1989) that economic mechanisms should not rely on precise details of the environment. Thereby, this paper falls in a recent literature in economics and computer science that aims at constructing mechanisms that work well under different distributions of valuations (e.g. Bergemann and Schlag, 2008, 2011; Dhangwatnotai et al., 2014; Roughgarden et al., 2012; Devanur et al., 2011; Fu et al., 2013, 2014). The second part of our paper is also closely related to a recent literature that analyzes the optimal use of a single sample in this context (Dhangwatnotai et al., 2014; Huang et al., 2014). Huang et al. (2014) showed that posting the sampled value as a take-it-or-leave-it price, which guarantees half of optimal revenue, is the optimal deterministic mechanism. We show in Section 4 that randomly inflating and shading the sample value can strictly improve the guarantee. In contrast to studying the use of a single sample, Cole and Roughgarden (2014) give the asymptotic number of samples needed for a given approximation factor, in the presence of asymmetric bidders. Finally, this avenue of study is related to a literature on parametric auctions, in which a mechanism can depend on limited statistical information about the valuation distributions (such as the median, mean, and/or variance) (Azar and Micali, 2012; Azar et al., 2013). The best-known parametric auctions for our setting are deterministic, but it is natural to ask whether randomization can help in this context.

\section{Preliminaries}

Single Item Auctions In a classical single item auction, the auctioneer has a single item to sell to $n$ bidders. Each bidder $i$ has a private value $v_{i}$ for receiving the item. We will consider the symmetric Bayesian setting, where each bidder's value is drawn independently from a distribution $F$ with density function $f=F^{\prime}$. We write $\boldsymbol{v}=\left(v_{1}, \ldots, v_{n}\right)$ for the profile of values. An auction consists of allocation rules $x_{i}: \mathbb{R}_{+}^{n} \rightarrow[0,1]$ and payment rules $p_{i}: \mathbb{R}_{+}^{n} \rightarrow \mathbb{R}$, meaning that, when the bid profile is $\boldsymbol{v}$, each bidder $i$ gets the item with probability $x_{i}(\boldsymbol{v})$ and makes a payment of $p_{i}(\boldsymbol{v})$. A bidder's utility is then $x_{i}(\boldsymbol{v}) v_{i}-p_{i}(\boldsymbol{v})$.

The allocation rules have to satisfy the feasibility constraint $\sum_{i} x_{i}(\boldsymbol{v}) \leq 1$, for all $\boldsymbol{v}$. We also require an auction to be individually rational, that is, bidders always get nonnegative returns. 
Formally, for each bidder $i$ and all bid profiles $\boldsymbol{v}, v_{i} x_{i}(\boldsymbol{v})-p_{i}(\boldsymbol{v}) \geq 0$. An auction is Bayesian incentive compatible if, for each bidder $i$ and value $v_{i}$, the bidder's expected utility (over randomness in other bidders' values) is maximized by bidding her true valuation. Formally, for each $i$, value $v_{i}$, and deviation $v_{i}^{\prime}$,

$$
\mathbf{E}_{v_{-i}}\left[x_{i}\left(v_{i}, v_{-i}\right) v_{i}-p_{i}\left(v_{i}, v_{-i}\right)\right] \geq \mathbf{E}_{v_{-i}}\left[x_{i}\left(v_{i}^{\prime}, v_{-i}\right) v_{i}-p_{i}\left(v_{i}^{\prime}, v_{-i}\right)\right] .
$$

For each bidder $i$, an allocation rule generates an interim allocation rule which maps her value $v_{i}$ to a winning probability, in expectation over the other bidders' bids. We abuse notation and use $x_{i}\left(v_{i}\right)=\mathbf{E}_{v_{-i}}\left[x_{i}\left(v_{i}, v_{-i}\right)\right]$ to denote the interim allocation rule. The interim payment rule $p_{i}\left(v_{i}\right)$ is similarly defined. Bayesian incentive compability is therefore easily expressed by the interim allocation rules and interim payment rules: for each bidder $i$, each value $v_{i}$ and possible deviation $v_{i}^{\prime}$, we have $x_{i}\left(v_{i}\right) v_{i}-p_{i}\left(v_{i}\right) \geq x_{i}\left(v_{i}^{\prime}\right) v_{i}-p_{i}\left(v_{i}^{\prime}\right)$.

The expected revenue of an auction is $\mathbf{E}_{\boldsymbol{v}}\left[\sum_{i} p_{i}(\boldsymbol{v})\right]$. Given a value distribution $F$, a revenue optimal auction is one whose expected revenue is optimal among all individually rational and Bayesian incentive compatible auctions. We refer to the revenue of this auction as the optimal revenue.

All auctions in this paper will satisfy the stronger (dominant strategy) incentive compatibility property, i.e., no matter what the the other bidders bid, it is always in a bidder's best interest to truthfully bid her value. Since we will only consider incentive compatible auctions (which is without loss of generality by the revelation principle (Mverson, 1981)), we have used the same symbol $v$ for values and bids. In general, an auction will be incentive compatible if each bidder faces a take-it-or-leave-it price that does not depend on her own bid. All auctions considered in this paper will obviously satisfy this condition?2

Revenue-Optimal Auctions In his seminal work, Myerson (1981) laid the foundation for the study of revenue-optimal auctions. The following theorem summarizes the part of his results that will be used in this work.

Theorem 1 (Myerson, 1981). In a single item auction where each bidder's value is drawn i.i.d. from a distribution $F$, for any Bayesian incentive compatible auction with interim allocation rules $x_{i}$ 's,

(i) The expected revenue from each bidder is equal to the virtual surplus, defined as

$$
\mathbf{E}_{v_{i} \sim F}\left[x_{i}\left(v_{i}\right) \cdot\left(v_{i}-\frac{1-F\left(v_{i}\right)}{f\left(v_{i}\right)}\right)\right] \text {. }
$$

The term $v_{i}-\frac{1-F\left(v_{i}\right)}{f\left(v_{i}\right.}$ is called the virtual value of the value $v_{i}$. In other words, the virtual surplus is the winning virtual value in expectation.

(ii) When the distribution $F$ is regular, i.e., when the virtual value is monotone nondecreasing with the value, the optimal auction is the second price auction with a reserve price $v_{*}$. In this auction, the item is only sold when at least one bid is above $v_{*}$, and the winner pays the higher of $v_{*}$ and the second highest bid.

\footnotetext{
${ }^{2}$ We introduced the notion of Bayesian incentive compatibility here because our benchmark optimal revenue mechanism needs only to satisfy Bayesian incentive compatibility. As Myerson showed, this is in fact equal to the revenue of the optimal dominant strategy incentive compatible auction.
} 
Bulow and Roberts (1989)'s Interpretation and Revenue Curves In a classic work, Bulow and Roberts (1989) gave an interpretation of Myerson (1981)'s optimal auciton and drew a connection between the theory of optimal auctions and the theory of monopolist price discrimination. This connection reveals much economic intuition underlying Myerson's results, and provides powerful technical tools. It is this viewpoint and tools that we will heavily use in this work, and we explain this connection in some detail here.

A bidder whose value is drawn from a distribution $F$ can be seen as a market where the customers' values are distributed according to $F$, which gives rise to its demand curve. In particular, if the monopolizer sets the price of a good to sell at $p$, then only customers whose value are above $p$ will buy the good. The demand is therefore $1-F(p)$. Each value $v$ is in this way mapped to its quantile $q(v):=1-F(v)$, its relative standing in this market. The revenue collected when the monopolizer sells a quantity $q$ is given by $R(q):=v(q) q$, where $v(q):=F^{-1}(1-q)$. This function $R:[0,1] \rightarrow \mathbb{R}_{+}$is called the revenue curve. Back from this analogy to the bidder, the quantile $q$ of a value $v$ is the probability with which the buyer will buy at a take-it-or-leave-it price of $v$. Note that for any distribution $F, q$ is uniformly distributed on $[0,1]$.

Bulow and Roberts (1989) showed that the slope of the revenue curve at a quantile $q$ is exactly equal to the virtual value of the value corresponding to $q$. Formally,

$$
R^{\prime}(q(v))=\left.\frac{\mathrm{d} R(q)}{\mathrm{d} q}\right|_{q=q(v)}=v-\frac{1-F(v)}{f(v)} .
$$

As a consequence, regular distributions, in which virtual values are monotone nondecreasing with values, are exactly the distributions with concave revenue curves. Also, for an auction with interim allocation rule $x_{i}$, the virtual surplus (equal to the expected revenue) from a bidder is given by $\int_{0}^{1} R^{\prime}(q) x_{i}(q(v)) \mathrm{d} q$.

The highest point of a revenue curve corresponds to the optimal revenue one could get by setting a take-it-or-leave-it price for a bidder. This price we call the monopoly reserve price and denote by $v_{*}$. We denote the quantile of $v_{*}$ by $q_{*}$.

Throughout the paper we will assume $R(0)=R(1)=0$. This is a rather standard assumption in the literature and is without loss of generality; for completeness we justify it in Appendix A.

\footnotetext{
Analysis of Second Price Auctions by Bulow and Klemperer (1996) Bulow and Klemperer (1996) showed that, for bidders whose values are drawn from a regular distribution, the revenue of an $n+1$ bidder second price auction without reserve price is always (weakly) better than the revenue of the $n$ bidder revenue optimal revenue auction. This immediately implies that for $n$ bidders, the revenue of the second price auction without a reserve price is at least $1-\frac{1}{n}$ of the optimal revenue.
}

\section{Inflated Second Price Auction}

We will consider a variant of the second price auction, which will sometimes inflate the bid of the second-highest bidder before offering that bid as a fixed price for the highest bidder.

Definition 1. The $\delta$-inflated second price auction offers the item to the highest bidder at a take-itor-leave-it price set as $(1+\delta)$ times the second highest bid. The $(\epsilon, \delta)$-inflated second price auction runs the $\delta$-inflated second price auction with probability $\epsilon$, and runs the second price auction with probability $1-\epsilon$. 
We will prove the following main theorem in this section.

Theorem 2. For any $n$, there is a $(\epsilon, \delta)$-inflated second price auction, such that for any $n$ bidders with values drawn i.i.d. from a regular distribution, the revenue of the inflated second price auction is strictly larger than $\frac{n-1}{n}$ fraction of the optimal revenue.

At the end of the section, we give the improved approximation ratios of 0.512 for the case $n=2$.

We will begin our analysis by studying the relationship between the optimal revenue and the revenue of the second price auction (SPA) without reserve. The goal of this analysis is to refine the standard $\frac{n}{n-1}$-approximation result and to present an approximation that depends on the quantile of the optimal reserve price, $q_{*}$.

We begin by deriving a bound on the additive revenue loss suffered by using SPA rather than the optimal auction.

Lemma 3. For $n$ bidders with values drawn i.i.d. from a regular distribution, the difference between the revenue of the optimal auction and that of the second price auction is at most $R\left(q_{*}\right)\left(1-q_{*}\right)^{n-1}$.

Proof. The optimal auction is a second price auction with a reserve at $v_{*}$. The allocation rules for the optimal auction and the second price auction therefore differ only when the highest value is below $v_{*}$, i.e., its corresponding quantile is larger than $q_{*}$. Such quantiles correspond to negative virtual values, and make up the difference between the optimal auction (where the contribution is zero) and the second price auction (where the contribution is negative). For a bidder bidding at quantile $q$, the probability that it is the lowest quantile is $(1-q)^{n-1}$. The total negative virtual surplus generated by one bidder, over quantiles above $q_{*}$, is therefore

$$
\begin{aligned}
& \int_{q_{*}}^{1} R^{\prime}(q)(1-q)^{n-1} \mathrm{~d} q \\
= & \left.R(q)(1-q)^{n-1}\right|_{q_{*}} ^{1}+(n-1) \int_{q_{*}}^{1} R(q)(1-q)^{n-2} \mathrm{~d} q \\
= & -R\left(q_{*}\right)\left(1-q_{*}\right)^{n-1}+(n-1) \int_{q_{*}}^{1} R(q)(1-q)^{n-2} \mathrm{~d} q
\end{aligned}
$$

This is the negative of the difference between the optimal revenue and the revenue of the second price auction. To get an upper bound of the difference, we need to find a lower bound for the integral. Using the concavity of $R(q)$, we know that, for $q \geq q_{*}, R(q) \geq R\left(q_{*}\right) \cdot \frac{1-q}{1-q_{*}}$. Therefore, we know that the quantity above is at most

$$
-R\left(q_{*}\right)\left(1-q_{*}\right)^{n-1}+(n-1) \int_{q_{*}}^{1} R\left(q_{*}\right) \frac{1-q}{1-q_{*}} \cdot(1-q)^{n-2} \mathrm{~d} q=-\frac{1}{n} R\left(q_{*}\right)\left(1-q_{*}\right)^{n-1} .
$$

This is the revenue difference due to each bidder. Multiplying this by $n$ gives us the lemma.

As a corollary, we obtain the following bound on the ratio between the revenue of SPA and the optimal revenue.

Corollary 1. For $n$ i.i.d. bidders with a regular valuation distribution, for any $q_{*} \in(0,1]$, the second price auction extracts at least a $\frac{1-\left(1-q_{*}\right)^{n-1}}{1-\left(1-q_{*}\right)^{n}}$ fraction of the optimal revenue. 
Proof. We give a lower bound on the optimal revenue, which, combined with Lemma 3, gives a lower bound on the approximation ratio of the SPA. The optimal auction could post $v_{*}$ as a takeit-or-leave-it price to each bidder in turn, and sell at that price to the first bidder who accepts. This gives a revenue of $R\left(q_{*}\right)\left[1+\left(1-q_{*}\right)+\left(1-q_{*}\right)^{2}+\cdots+\left(1-q_{*}\right)^{n-1}\right]$. The ratio of the revenue of the second price auction to the optimal revenue is therefore at least

$$
1-\frac{\left(1-q_{*}\right)^{n-1}}{1+\left(1-q_{*}\right)+\cdots+\left(1-q_{*}\right)^{n-1}}=\frac{1+\left(1-q_{*}\right)+\cdots+\left(1-q_{*}\right)^{n-2}}{1+\left(1-q_{*}\right)+\cdots+\left(1-q_{*}\right)^{n-1}}=\frac{1-\left(1-q_{*}\right)^{n-1}}{1-\left(1-q_{*}\right)^{n}} .
$$

Note that the factor in Corollary 1 is a strictly increasing function in $q_{*}$, and is equal to $\frac{n-1}{n}$ when $q_{*}=0$. For the case $n=2$, we see that the approximation ratio of the SPA is at least $\frac{1}{2-q_{*}}$.

We will now analyze the $\delta$-inflated second price auction, and show that its approximation ratio is better when $q_{*}$ is small. Before that, we first prove a technical lemma that gives us bounds on quantiles in a regular distribution for values that are apart by a multiplicative factor.

Lemma 4. For any regular distribution:

1. $q\left(\frac{v_{*}}{1+\delta}\right) \leq(1+\delta) q_{*}$, and

2. For any $\tilde{q} \geq q_{*}, q\left(\frac{v(\tilde{q})}{1+\delta}\right) \geq \frac{1+\delta}{1+\delta \tilde{q}} \tilde{q}$.

Proof. The first statement is a direct consequence of the optimality of $v_{*}$ as a reserve price for a single bidder: $v_{*} q_{*}=R\left(q_{*}\right) \geq R\left(q\left(v_{*} /(1+\delta)\right)\right)=q\left(v_{*} /(1+\delta)\right) \cdot v_{*} /(1+\delta)$.

For the second statement, let us denote by $q_{1}$ the quantile of $v(\tilde{q}) /(1+\delta)$. By regularity of the distribution, the revenue curve is concave, and because $q_{1}$ is greater than $\tilde{q}$ and both are greater than $q_{*}$, on the revenue curve the point $\left(q_{1}, R\left(q_{1}\right)\right)$ is above the straight line connecting $(1, R(1)=0)$ and $(\tilde{q}, R(\tilde{q}))$. Therefore,

$$
\frac{R(\tilde{q})}{1-\tilde{q}} \leq \frac{R\left(q_{1}\right)}{1-q_{1}}, \quad \Rightarrow \quad \frac{v(\tilde{q}) \tilde{q}}{1-\tilde{q}} \leq \frac{q_{1} v(\tilde{q})}{(1+\delta)\left(1-q_{1}\right)}
$$

Rearranging the terms we have that $q_{1} \geq \frac{1+\delta}{1+\delta \tilde{q}} \tilde{q}$.

The next lemma lower bounds the approximation ratio of $\delta$-inflated SPA for distributions with a small $q_{*}$.

Lemma 5. For a regular distribution with $q_{*}<1 / n$, the revenue of the $\delta$-inflated SPA for $n$ i.i.d. bidders is at least

$$
n R\left(q_{*}\right)\left[\left[1-(1+\delta) q_{*}\right]^{n-1}-\left(1-\frac{(1+\delta) q_{*}}{1+\delta q_{*}}\right)^{n-1}+\frac{(n-1)(1+\delta)}{1-q_{*}} \int_{q_{*}}^{1} \frac{(1-q)^{n-1}}{(1+\delta q)^{n}} \mathrm{~d} q\right] .
$$

Proof. Let the interim allocation rule of the $\delta$-inflated second price auction for a bidder $i$ be $x_{i}$. Recall that the revenue from a bidder $i$ is given by $\int_{0}^{1} R^{\prime}(q) x_{i}(q) \mathrm{d} q$. On $\left[0, q_{*}\right], R^{\prime}(q)$ is positive, and we need to lower bound $x_{i}(q)$; on $\left(q_{*}, 1\right], R^{\prime}(q)$ is negative, and we need to upper bound $x_{i}(q)$. 
We first lower bound $\int_{0}^{q_{*}} R^{\prime}(q) x_{i}(q) \mathrm{d} q$. For such quantiles, the corresponding value is larger than $v_{*}$. Obviously, if any bidder has a value $v>v_{*}$, as long as all other bidders' values are below $\frac{v_{*}}{1+\delta}$, the bidder bidding $v$ will win. By Lemma 4, the quantile of $\frac{v_{*}}{1+\delta}$ is at most $(1+\delta) q_{*}$, and therefore the probability that a bidder's value is below $\frac{v_{*}}{1+\delta}$ is at least $1-(1+\delta) q_{*}$. Hence, the expected revenue collected from each bidder for values larger than the monopoly reserve is at least

$$
\int_{0}^{q_{*}} R^{\prime}(q)\left[1-(1+\delta) q_{*}\right]^{n-1} \mathrm{~d} q=\left[1-(1+\delta) q_{*}\right]^{n-1} R\left(q_{*}\right) .
$$

We then upper bound the negative of $\int_{q_{*}}^{1} R^{\prime}(q) x_{i}(q) \mathrm{d} q$. For such quantiles, the corresponding value is smaller than $v_{*}$. Recall that we would like to upper bound $x_{i}(q)$. By the definition of $\delta$-inflated SPA, such a value wins the auction if and only if all other bidders bid below $v /(1+\delta)$. By Lemma 4, the quantile of $v /(1+\delta)$ is at least $\frac{1+\delta}{1+\delta q} q$. In other words, the probability that an independent draw has value less than $\frac{v}{1+\delta}$ is at most $1-\frac{1+\delta}{1+\delta q} q=\frac{1-q}{1+\delta q}$. Therefore, the negative contribution to the virtual surplus by each bidder is lower bounded by

$$
\begin{aligned}
\int_{q_{*}}^{1} R^{\prime}(q)\left(\frac{1-q}{1+\delta q}\right)^{n-1} \mathrm{~d} q & =\left.R(q)\left(\frac{1-q}{1+\delta q}\right)^{n-1}\right|_{q_{*}} ^{1}+(n-1) \int_{q_{*}}^{1} R(q)\left(\frac{1-q}{1+\delta q}\right)^{n-2} \cdot \frac{1+\delta}{(1+\delta q)^{2}} \mathrm{~d} q \\
& =-R\left(q_{*}\right)\left(\frac{1-q_{*}}{1+\delta q_{*}}\right)^{n-1}+(n-1)(1+\delta) \int_{q_{*}}^{1} R(q) \frac{(1-q)^{n-2}}{(1+\delta q)^{n}} \mathrm{~d} q,
\end{aligned}
$$

where in the first step we did an integration by part, and in the second step we used the fact $R(1)=0$. Since we aim to lower bound this quantity, we use the fact that $R(q)$ to the right of $q_{*}$ is pointwise lower bounded by $R\left(q_{*}\right) \cdot \frac{1-q}{1-q_{*}}$ because of its concavity. Substituting this, we have that

$$
\int_{q_{*}}^{1} R(q) \frac{(1-q)^{n-2}}{(1+\delta q)^{n}} \mathrm{~d} q \geq \int_{q_{*}}^{1} R\left(q_{*}\right) \cdot \frac{1-q}{1-q_{*}} \cdot \frac{(1-q)^{n-2}}{(1+\delta q)^{n}} \mathrm{~d} q=\frac{R\left(q_{*}\right)}{1-q_{*}} \int_{q_{*}}^{1} \frac{(1-q)^{n-1}}{(1+\delta q)^{n}} \mathrm{~d} q .
$$

Combining everything together, the revenue of the $\delta$-inflated second price auction is at least

$$
n R\left(q_{*}\right)\left[\left[1-(1+\delta) q_{*}\right]^{n-1}-\left(1-\frac{(1+\delta) q_{*}}{1+\delta q_{*}}\right)^{n-1}+\frac{(n-1)(1+\delta)}{1-q_{*}} \int_{q_{*}}^{1} \frac{(1-q)^{n-1}}{(1+\delta q)^{n}} \mathrm{~d} q\right]
$$

Lemma 6. For $\delta=1$ and $q_{*}<\frac{1}{n}$, the ratio of the 1 -inflated second price auction revenue to the optimal revenue is at least

$$
\begin{aligned}
& {\left[1-(1+\delta) q_{*}\right]^{n-1}-\left(1-\frac{(1+\delta) q_{*}}{1+\delta q_{*}}\right)^{n-1}+\frac{(n-1)\left(1-q_{*}\right)^{n-1}}{n\left(1+q_{*}\right)^{n}}} \\
& +\frac{n-1}{n\left(1-q_{*}\right)}\left[\left(1-\frac{1}{n^{2}}\right)^{-n}-\frac{1}{\left(1-q_{*}^{2}\right)^{n}}\right]\left(1-\frac{1}{n}\right)^{2 n} \cdot
\end{aligned}
$$

We relegate the proof to Appendix B. 
Proof of Theorem 2. By Lemma 6, for $q_{*}=0$, the approximation ratio of the 1-inflated second price auction is at least $\frac{n-1}{n} \cdot\left[1+\left(1-\frac{2}{n+1}\right)^{n}\right]$, which is strictly greater than $\frac{n-1}{n}$ for any $n$. Since the bound given by Lemma 6 is a continuous function, for a sufficiently small $\underline{q}>0$, for all $q_{*}<\underline{q}$, the 1 -inflated SPA has an approximation ratio at least $\frac{n-1}{n} \cdot\left[1+\frac{1}{2}\left(1-\frac{2}{n+1}\right)^{n}\right]$. Recall that the approximation ratio we derived in Corollary 1 is a strictly increasing function in $q_{*}$ that equals to $\frac{n-1}{n}$ at $q_{*}=0$. Therefore at $\underline{q}$, the approximation ratio of the SPA is $\frac{n-1}{n}(1+\gamma)$ for some $\gamma>0$. Taking $\epsilon>0$ small enough such that $(1+\gamma)(1-\epsilon)>1+\eta$ for some $\eta>0$, we will ensure that for all $q_{*}>\underline{q}$, the approximation of the $(\epsilon, \delta)$-inflated SPA is at least $\frac{n-1}{n}(1+\eta)$ (this pessimistically does not assume any revenue coming from the 1-inflated SPA). On the other hand, for $q_{*} \leq \underline{q}$, since the approximation ratio of the SPA is always at least $\frac{n-1}{n}$, the $(\epsilon, \delta)$-inflated SPA has an approximation ratio at least $\frac{n-1}{n}\left(1+\frac{\epsilon}{2}\left(1-\frac{2}{n+1}\right)^{n}\right)$. This proves the theorem.

Remark 1. Lemma 6 is in place because the integral in (1) is not easy for general values of $n$. The argument in the proof of Theorem 2 is rather pessimistic. However, for concrete values of $n$, one can compute the revenue lower bound in Lemma 5 for any $\delta>0$, without going through further losses in the analysis of Lemma 6 and Theorem 2, and get better approximation ratios. For example, for $n=2$ and $\delta=1$,

$$
\int_{q_{*}}^{1} \frac{(1-q)^{n-1}}{(1+\delta q)^{n}} \mathrm{~d} q=\int_{q_{*}}^{1} \frac{1-q}{(1+q)^{2}} \mathrm{~d} q=\frac{2}{q_{*}+1}+\log \left(\frac{q_{*}+1}{2}\right)-1 .
$$

One can substitute this into (2) and combine it with Corollary 1 numerical computation shows that the $(0.15,1)$-inflated second price auction gives at least 0.512 fraction of the optimal revenue.

\section{Revenue Maximization with a Single Sample}

In this section we show that, for any buyer with value drawn from a regular distribution $F$, with one sample from the same distribution, one can extract strictly more than half of the the optimal revenue, by introducing randomization in the use of the sample. We denote the sample by $s$, and the buyer's value by $v$. Note that in this setting, the optimal revenue is $\operatorname{simply} R\left(q_{*}\right)$.

Definition 2. The post-the-sample algorithm posts the sample $s$ as a take-it-or-leave-it price. The $\rho$-shaded post-the-sample algorithm posts $(1-\rho) s$ as a take-it-or-leave-it price. The $\delta$-inflated post-the-sample algorithm posts $(1+\delta) s$ as the take-it-or-leave-it price. The $(\zeta, \rho, \epsilon, \delta)$-randomized post-the-sample algorithm runs $\rho$-shaded post-the-sample with probaiblity $\zeta, \delta$-inflated post-thesample with probability $\epsilon$, and (normal) post-the-sample with probability $1-\zeta-\epsilon$.

Theorem 7. There exists $\zeta, \rho, \epsilon, \delta$ such that for any regular distribution, with a single sample, the $(\zeta, \rho, \epsilon, \delta)$-randomized post-the-sample algorithm extracts strictly more than half of the optimal revenue.

Using our randomized post-the-sample algorithm in place of the original post-the-sample algorithm in the auction of Dhangwatnotai et al. (2014), we have the following corollary.

Corollary 2. In a single-item multi-bidder auction, where bidders' values are drawn independently from regular distributions, and where for each bidder there is at least another bidder whose value is drawn from the same distribution, there is a prior-independent auction whose revenue is strictly better than $\frac{1}{8}$ of the revenue of the optimal auction which knows all distributions. 
In Theorem 7, the improvement over the one half approximation will be on the order of $10^{-8}$, and this is admittedly mainly of theoretical interest (we also made no effort in optimizing the parameters). The concrete values of $\zeta$ and $\epsilon$ are given in the proof near the end of the section. We first analyze the performance of the three ingredient mechanism in the randomized post-the-sample algorithm, given in the next three lemmas.

Lemma 8. For any $q_{*} \in\left[0, \frac{1}{2}\right]$, the approximation ratio of the 1 -inflated post-the-sample algorithm is at least

$$
-2 q_{*}+\frac{2 q_{*}}{1+q_{*}}+\frac{2}{1-q_{*}}\left[\frac{2}{q_{*}+1}+\log \left(\frac{q_{*}+1}{2}\right)-1\right] .
$$

Proof. The revenue from a single bidder by posting twice the sample is exactly half of the revenue from a two bidder 1-inflated second price auction where the two bidders values are drawn i.i.d. from the same regular distribution. The lemma then follows from Lemma 5 and Remark 1.

Lemma 9. For any regular distribution, if posting a price of $\frac{v_{*}}{1-\rho}$ obtains a revenue that is $\beta$ fraction of the optimal revenue $R\left(q_{*}\right)$, then the revenue of the $\rho$-shaded post-the-sample algorithm is at least

$$
\left[(1-\rho)\left(\frac{q_{*}+1}{2}-\beta q_{*}\right)+q_{*} \beta \rho\right] R\left(q_{*}\right) .
$$

This is the most technically involved proof of this section. We relegate it to Appendix C.

Lemma 10. If for a regular distribution, posting a price of $\frac{v_{*}}{1-\rho}$ obtains a revenue that is $\beta$ fraction of the optimal revenue $R\left(q_{*}\right)$, then the revenue of the post-the-sample algorithm is at least $\frac{1}{2}(1+$ $\left.q_{*} \rho \beta\right) R\left(q_{*}\right)$.

Proof. The revenue of the post-the-sample algorithm is equal to the total area under the revenue curve (Dhangwatnotai et al., 2014). We will therefore give a lower bound on this area. In general, subject to concavity and fixing $R\left(q_{*}\right)$, the area under the revenue curve is minimized at $\frac{1}{2} R\left(q_{*}\right)$ by the triangle. Given $\beta$, the fraction of $R\left(q_{*}\right)$ extracted by posting a price $v_{*} /(1-\rho)$, the quantile of $v_{*} /(1-\rho)$ is given by $\beta R\left(q_{*}\right)(1-\rho)$. At this quantile, the triangle revenue curve would have a revenue of $R\left(q_{*}\right) \beta(1-\rho)$, but the current revenue is $R\left(q_{*}\right) \beta$. The two differ by $\rho \beta R\left(q_{*}\right)$. The extra area over the triangle is therefore at least $\frac{1}{2} q_{*} \rho \beta R\left(q_{*}\right)$.

Proof of Theorem 7. We will show that setting $\delta=1, \rho=0.01$, and sufficiently small $\epsilon$ and $\zeta$, with $\epsilon=4 \zeta$, will guarantee a revenue better than $\frac{1}{2}$ of the optimal revenue. Note that (4) is a decreasing function in $\beta$ for $\rho<0.5$. For $q_{*} \leq 0.02$ and any value of $\beta, 0.8 \times(4)+0.2 \times(3)$ is at least $0.505 R\left(q_{*}\right)$, by taking the worst value of $\beta=1$. For $\beta \leq 0.05$ and any value of $q_{*}$, $0.8 \times(4)+0.2 \times(3)$ is at least $0.518 R\left(q_{*}\right)$, by taking the worst value of $\beta=0.05$, and the minimum is taken at $q_{*}=0$. For the only remaining case, i.e., $q_{*}>0.02$ and $\beta>0.05$, Lemma 10 gives that the revenue of the post-the-sample algorithm is at least $0.500005 R\left(q_{*}\right)$. Therefore, running post-the-sample with probability $1-10^{-6}$ guarantees a revenue of better than $0.5+10^{-6}$ fraction of $R\left(q_{*}\right)$ in this case. With the remaining probability of $10^{-6}$, running the $\delta$-inflated post-the-sample with probability $2 \times 10^{-7}$ and the $\rho$-shaded post-the-sample with probability $8 \times 10^{-7}$ guarantees a revenue of $0.5+5 \times 10^{-9}$ fraction of the optimal revenue in the cases analyzed above. Overall, 
the $\left(0.8 \times 10^{-6}, 0.01,0.2 \times 10^{-6}, 1\right)$-randomized post-the-sample algorithm gives an approximation ratio of at least $0.5+5 \times 10^{-9}$.

\section{References}

Azar, P., Daskalakis, C., Micali, S., and Weinberg, S. M. (2013). Optimal and efficient parametric auctions. In Proceedings of the Twenty-Fourth Annual ACM-SIAM Symposium on Discrete Algorithms, pages 596-604. SIAM.

Azar, P. and Micali, S. (2012). Optimal parametric auctions. Mit-csail-tr-2012-011.

Bergemann, D. and Schlag, K. (2011). Should i stay or should i go? search without priors. mimeo, available at: http://www.gtcenter.org/Archive/2011/Conf/Schlag1314. pdf (last accessed 18 April 2014).

Bergemann, D. and Schlag, K. H. (2008). Pricing without priors. Journal of the European Economic Association, 6(2-3):560-569.

Bulow, J. and Klemperer, P. (1996). Auctions versus negotiations. The American Economic Review, pages 180-194.

Bulow, J. and Roberts, J. (1989). The simple economics of optimal auctions. Journal of Political Economy, 97(5):1060-1090.

Cole, R. and Roughgarden, T. (2014). The sample complexity of revenue maximization. In Symposium on Theory of Computing, STOC 2014, New York, NY, USA, May 31 - June 03, 2014, pages $243-252$.

Devanur, N. R., Hartline, J. D., Karlin, A. R., and Nguyen, C. T. (2011). Prior-independent multi-parameter mechanism design. In WINE, pages 122-133.

Dhangwatnotai, P., Roughgarden, T., and Yan, Q. (2014). Revenue maximization with a single sample. Games and Economic Behavior.

Fu, H., Haghpanah, N., Hartline, J. D., and Kleinberg, R. (2014). Optimal auctions for correlated buyers with sampling. In ACM Conference on Economics and Computation, EC '14, Stanford, CA, USA, June 8-12, 2014, pages 23-36.

Fu, H., Hartline, J. D., and Hoy, D. (2013). Prior-independent auctions for risk-averse agents. In ACM Conference on Electronic Commerce, pages 471-488.

Huang, Z., Mansour, Y., and Roughgarden, T. (2014). Making the most of your samples. arXiv preprint arXiv:1407.2479.

Myerson, R. (1981). Optimal auction design. Mathematics of Operations Research, 6(1):pp. 58-73.

Roughgarden, T., Talgam-Cohen, I., and Yan, Q. (2012). Supply-limiting mechanisms. In ACM Conference on Electronic Commerce, pages 844-861. 
Wilson, R. B., . (1989). Game-theoretic approaches to trading processes. In Advances in economic theory. Cambridge University Press.

\section{A Missing Argument from Section 2}

Throughout the paper we have made the assumption $R(0)=R(1)=0$. This is standard in the literature, and we briefly show that this is without loss of generality.

A value distribution whose support includes 0 satisfies $R(1)=0$; otherwise, one can mix into the distribution with probability $\epsilon$ a uniform distribution on $[0, \underline{v}]$, where $\underline{v}$ is the infimum of the support. As $\epsilon$ approaches 0, this mixture converges to the original distribution, and for sufficiently small $\epsilon$, the resulting revenue curve remains concave if the original revenue curve is. As for the assumption $R(0)=0$, we first justify it for bounded support distributions. When there is no point mass on the supremum of the support, its revenue will be 0 at quantile 0 . If this is not the case, we can mix into the distribution with probability $\epsilon$ a uniform distribution on $[\bar{v}, \bar{v}+\delta]$, where $\bar{v}$ is the supremum of the support, and $\delta>0$ is a small positive real number. For sufficiently small $\delta$ and $\epsilon$, the resulting revenue curve will be concave if the original one is; as $\epsilon$ approaches 0 , the mixture also converges to the original distribution. Any unbounded distribution's revenue can be approached arbitrarily well by taking its truncation at higher and higer values. The truncated distribution is bounded. All our analysis would not be affected by all such asymptotic approximations, and we will simply assume $R(0)=R(1)=0$.

\section{B Missing Proofs from Section 3}

Proof of Lemma [6. We estimate the integral in (10. For $\delta=1$,

$$
\begin{aligned}
\int_{q_{*}}^{1} \frac{(1-q)^{n-1}}{(1+\delta q)^{n}} \mathrm{~d} q & =\int_{q_{*}}^{1} \frac{(1-q)^{n-1}}{(1+q)^{n}} \mathrm{~d} q=\int_{q_{*}}^{1} \frac{(1-q)^{2 n-1}}{\left(1-q^{2}\right)^{n}} \mathrm{~d} q \\
& =\int_{q_{*}}^{\frac{1}{n}} \frac{(1-q)^{2 n-1}}{\left(1-q^{2}\right)^{n}} \mathrm{~d} q+\int_{\frac{1}{n}}^{1} \frac{(1-q)^{2 n-1}}{\left(1-q^{2}\right)^{n}} \mathrm{~d} q \\
& \geq \frac{1}{\left(1-q_{*}\right)^{n}} \int_{q_{*}}^{\frac{1}{n}}(1-q)^{2 n-1} \mathrm{~d} q+\int_{\frac{1}{n}}^{1} \frac{(1-q)^{2 n-1}}{\left(1-\frac{1}{n^{2}}\right)^{n}} \mathrm{~d} q \\
& =\frac{\left(1-q_{*}\right)^{n}}{2 n\left(1+q_{*}\right)^{n}}+\frac{1}{2 n}\left[\left(1-\frac{1}{n^{2}}\right)^{-n}-\frac{1}{\left(1-q_{*}^{2}\right)^{n}}\right]\left(1-\frac{1}{n}\right)^{2 n} .
\end{aligned}
$$

Substituting this into (2), and noting that the optimal revenue is at most $n R\left(q_{*}\right)$, we obtain the lemma.

\section{Missing Proofs from Section 4}

Proof of Lemma 9. We first introduce some notations. Let $\varphi:[0,1] \rightarrow[0,1]$ be a function that maps a quantile $\tilde{q}$ to the quantile whose value is $1 /(1-\rho)$ times the value corresponding to $\tilde{q}$. 
(Formally, $\varphi(\tilde{q})=q\left(F^{-1}(1-\tilde{q}) /(1-\rho)\right)$ 草)

We consider the difference of $\rho$-shaded post-the-sample algorithm as compared with the plain post-the-sample algorithm. The two algorithms differ only when $s$ falls in the interval $\left[v, \frac{v}{1-\rho}\right]$ : the post-the-sample algorithm does not serve, whereas the $\rho$-shaded post-the-sample algorithm does. Conditioning on $v$, this event happens with probability $q(v)-\varphi(q(v))$. The revenue of the post-thesample algorithm is $\int_{0}^{1} R^{\prime}(q)(1-q) \mathrm{d} q$, and therefore the revenue of the $\rho$-shaded post-the-sample algorithm is

$$
\int_{0}^{1} R^{\prime}(q)(1-q) \mathrm{d} q+\int_{0}^{1} R^{\prime}(q)\left(q-\varphi(q) \mathrm{d} q=\int_{0}^{1} R^{\prime}(q) \mathrm{d} q-\int_{0}^{1} R^{\prime}(q) \varphi(q) \mathrm{d} q=-\int_{0}^{1} R^{\prime}(q) \varphi(q) \mathrm{d} q .\right.
$$

We need to lower bound this term.

When the buyer's value $v$ is smaller than $v_{*}$, its virtual value is negative. Here we would like to lower bound $\varphi(q)$. Let $\gamma$ be the ratio of the revenue of posting $v_{*}(1-\rho)$ to the optimal revenue $R\left(q_{*}\right)$. Let $q_{\gamma}$ be the quantile of the value $v_{*}(1-\rho)$, then because $v_{*}(1-\rho) q_{\gamma}=\gamma v_{*} q_{*}$, we have $q_{\gamma}=\gamma q_{*} /(1-\rho)$. For $v \leq v_{*}(1-\rho)$, we know that the revenue of posting the price $v$ is smaller than that of posting $v /(1-\rho)$. In other words, $v q(v) \leq \frac{v}{1-\rho} \varphi(q(v))$, and therefore we get the lower bound $\varphi(q) \geq(1-\rho) q, \forall q \in\left[q_{\gamma}, 1\right]$.

Let the revenue of posting $\frac{v_{*}}{1-\rho}$ be $\beta R\left(q_{*}\right)$. Then the quantile of the value $\frac{v_{*}}{1-\rho}$ is $\beta q_{*}(1-\rho)$. We know that, for all $q \in\left[q_{*}, q_{\gamma}\right], \varphi \geq \beta q_{*}(1-\rho)$. Now we have that

$$
\begin{aligned}
& -\int_{0}^{1} R^{\prime}(q) \varphi(q) \mathrm{d} q \geq-\int_{0}^{q_{*}} R^{\prime}(q) \varphi(q) \mathrm{d} q-\int_{q_{*}}^{q_{\gamma}} R^{\prime}(q) \beta q_{*}(1-\rho) \mathrm{d} q-(1-\rho) \int_{q_{\gamma}}^{1} R^{\prime}(q) q \mathrm{~d} q \\
= & -\int_{0}^{q_{*}} R^{\prime}(q) \varphi(q) \mathrm{d} q-\left.\beta q_{*}(1-\rho) R(q)\right|_{q_{*}} ^{q_{\gamma}}-(1-\rho)\left[\left.R(q) q\right|_{q_{\gamma}} ^{1}-\int_{q_{\gamma}}^{1} R(q) \mathrm{d} q\right]
\end{aligned}
$$

Using $R\left(q_{\gamma}\right)=\gamma R\left(q_{*}\right)$, this is equal to

$$
\begin{aligned}
& -\int_{0}^{q_{*}} R^{\prime}(q) \varphi(q) \mathrm{d} q-(1-\rho)\left[\beta q_{*}(\gamma-1) R\left(q_{*}\right)-\gamma R\left(q_{*}\right) \cdot \frac{\gamma q_{*}}{1-\rho}-\int_{q_{\gamma}}^{1} R(q) \mathrm{d} q\right] \\
= & -\int_{0}^{q_{*}} R^{\prime}(q) \varphi(q) \mathrm{d} q+(1-\rho) \beta q_{*}(1-\gamma) R\left(q_{*}\right)+\gamma^{2} q_{*} R\left(q_{*}\right)+(1-\rho) \int_{q_{\gamma}}^{1} R(q) \mathrm{d} q \\
\geq & -\int_{0}^{q_{*}} R^{\prime}(q) \varphi(q) \mathrm{d} q+(1-\rho) \beta q_{*}(1-\gamma) R\left(q_{*}\right)+\gamma^{2} q_{*} R\left(q_{*}\right)+(1-\rho) \gamma R\left(q_{*}\right) \cdot \frac{1}{2}\left(1-\frac{\gamma q_{*}}{1-\rho}\right) \\
= & -\int_{0}^{q_{*}} R^{\prime}(q) \varphi(q) \mathrm{d} q+(1-\rho) \beta q_{*}(1-\gamma) R\left(q_{*}\right)+\gamma^{2} q_{*} R\left(q_{*}\right)+\frac{\gamma R\left(q_{*}\right)}{2}\left(1-\rho-\gamma q_{*}\right),
\end{aligned}
$$

where in the inequality we used the concavity of $R$ and the fact $R\left(q_{\gamma}\right)=\gamma R\left(q_{*}\right)$. Note that the first term is a quantity unaffected by the value of $\gamma$. Therefore we can take the partial derivative of this lower bound with respect to $\gamma$ and get

$$
R\left(q_{*}\right)\left[-(1-\rho) \beta q_{*}+q_{*} \gamma+\frac{1-\rho}{2}\right] .
$$

\footnotetext{
${ }^{3}$ If $v /(1-\rho)$ is beyond the support of the distribution, just let $\varphi$ be 0 .
} 
Since $q_{\gamma} \geq q_{*}, v_{*}(1-\rho) q_{\gamma} \geq(1-\rho) v_{*} q_{*}$, and hence $\gamma \geq 1-\rho 4$ Therefore the partial derivative is lower bounded by

$$
R\left(q_{*}\right)(1-\rho)\left(q_{*}(1-\beta)+\frac{1}{2}\right) \geq 0 .
$$

Therefore, to minimize the revenue, the adversary should minimize $\gamma$. Hence, substituting $\gamma$ by $1-\rho$, we get the following lower bound on the revenue:

$$
-\int_{0}^{q_{*}} R^{\prime}(q) \varphi(q) \mathrm{d} q+\left[\frac{(1-\rho)\left(q_{*}+1\right)}{2}+q_{*} \beta \rho\right] R\left(q_{*}\right) .
$$

Now we consider bounding the first term. For $q \leq q_{*}$, its virtual value $R^{\prime}(q)$ is positive, therefore we need to upper bound $\varphi(q)$.

Recall that $\beta$ is the number such that the revenue of posting $\frac{v_{*}}{1-\rho}$ is $\beta R\left(q_{*}\right)$. Let $q_{1}$ denote the quantile of the value $v_{*} /(1-\rho)$, and we knew that $q_{1}=\beta q_{*}(1-\rho)$. Notice that, for every $q \in\left[0, q_{*}\right]$, $\varphi(q) \geq q_{1}$. We have

$$
\int_{0}^{q_{*}} R^{\prime}(q) \varphi(q) \mathrm{d} q \leq q_{1} \int_{0}^{q_{*}} R^{\prime}(q) \mathrm{d} q=\beta q_{*}(1-\rho) R\left(q_{*}\right) .
$$

Substituting this to (5), we see that the revenue is lower bounded by

$$
\left[(1-\rho)\left(\frac{q_{*}+1}{2}-\beta q_{*}\right)+q_{*} \beta \rho\right] R\left(q_{*}\right) .
$$

\footnotetext{
${ }^{4}$ A tighter lower bound for $\gamma$ would be $\frac{1-\rho}{1-\rho+\rho q_{*}}$, but the loose bound $1-\rho$ will suffice for our purpose.
} 